\title{
Enhancement of Cross-Borehole Pulse Radar Signature on a Partially Water-Filled Tunnel
}

\author{
Ji-Hyun Jung and Se-Yun Kim \\ Imaging Media Research Center, Korea Institute of Science and Technology, Hwarang-ro 14-gil 5, Seongbuk-gu, \\ Seoul 136-791, Republic of Korea
}

Correspondence should be addressed to Se-Yun Kim; ksy@imrc.kist.re.kr

Received 1 September 2014; Accepted 21 November 2014; Published 16 December 2014

Academic Editor: Matteo Pastorino

Copyright (C) 2014 J.-H. Jung and S.-Y. Kim. This is an open access article distributed under the Creative Commons Attribution License, which permits unrestricted use, distribution, and reproduction in any medium, provided the original work is properly cited.

\begin{abstract}
Cross-borehole pulse radar has been employed to detect a deeply located empty tunnel. In this paper, effects of underground water collected in the bottom of an empty tunnel on cross-borehole pulse radar signatures are analyzed numerically. B-scan images, stacks of received pulses, are calculated by applying the finite-difference time-domain (FDTD) method for 6 different heights of water from the bottom to the half height inside an empty tunnel. The most important features of an empty tunnel, the fastest time of peak (TOP) and time of arrival (TOA) extracted from the B-scan images, are slowed considerably depending on the increased height of water inside the tunnel. To compensate the weak TOP like that of an empty tunnel, a relation curve is formulated only utilizing measurable parameters of the fastest TOP and the fastest TOA. Then, a unified curve including the effects of two granites with the low and high dielectric properties is derived to cover widely varied dielectric properties of underground rocks. Based on the fastest TOP of an empty tunnel, the average difference between the fastest TOP of an empty tunnel and that of a partially water-filled tunnel decreases from $22.92 \%$ to $2.59 \%$ after enhancement.
\end{abstract}

\section{Introduction}

Two types of cross-borehole radars have been operated for detecting subversive intrusion tunnels deeply located in Korea since 1980s. One type is stepped-frequency continuous-wave (CW) radar characterized by the double-dip pattern at some specific frequencies in received signals [1-6]. Cross-borehole CW radar played a key role in detecting the Fourth Tunnel in Korea $[2,4]$. The other type is pulse radar [7-15], characterized by fast arrival in received pulse near the depth of an empty tunnel. Recently, cross-borehole pulse radar was developed in our group and its detectability of an empty tunnel was confirmed successfully in a well-suited tunnel test site in Korea [13-15]. However, underground water oozed out of underground rock can be collected in the bottom of an empty tunnel owing to highly weathered, jointed, and fractured underground rock of Korea. To detect such a partially water-filled tunnel, it should be analyzed properly whether conventional signatures of an empty tunnel can be employed immediately or not.
The pulse waveforms scattered by a fully water-filled cylindrical tunnel were already calculated analytically using 3-dimensional Green's function [16]. As expected, the first positive peak arrived latest in the central position of the tunnel. TE and TM scattering by a tunnel half-full of water were calculated in the presence of an air-earth interface using the 2-dimensional method of moment [17]. Imaging of scatterer buried in a lossy half space was developed using a regularizing procedure based on two-step inexact-Newton method [18]. A method to detect buried multilayer dielectric cylinders with elliptic cross-sections was analyzed using a global optimization procedure called memetic algorithm [19]. Until now, however, cross-borehole pulse radar signatures considering various height of collected water inside an empty tunnel are not available. Hence, experiments at a real test site in Korea can be considered utilizing our cross-borehole pulse radar. However, the safety of many visitors will render it nearly impossible to fill the in-site tunnel with water. Technically, the precise control of the water level in the tunnel is also very difficult since water is continuously oozed 


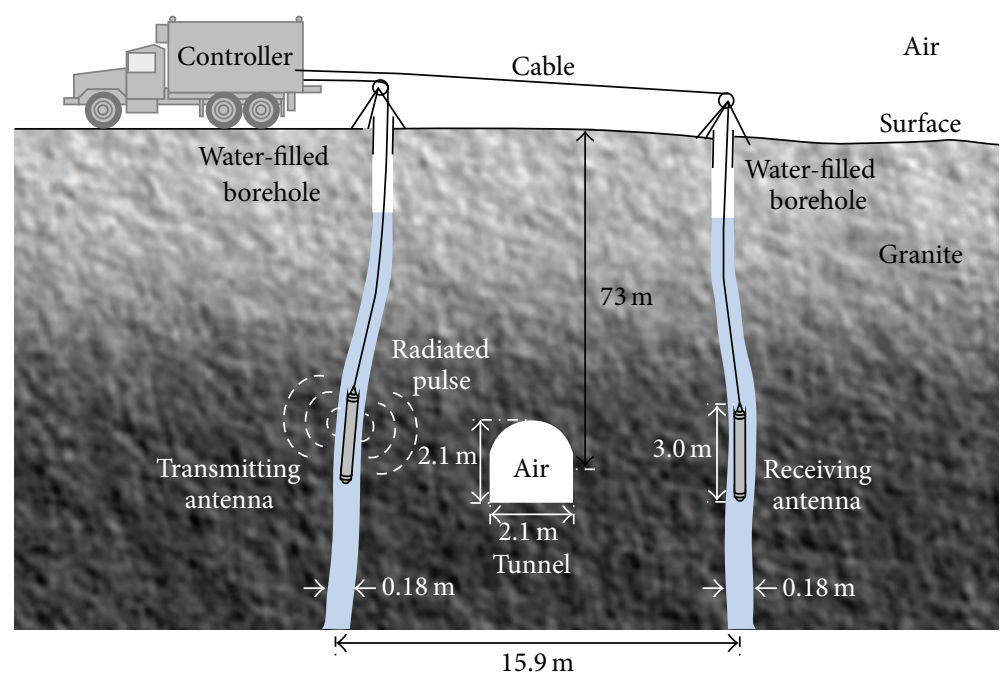

FIGURE 1: Experimental configuration of cross-borehole pulse radar in a well-suited tunnel test site in Korea.

out or permeated into the underground rock. It leads us to consider numerical simulations on a partially water-filled tunnel instead of field experiments. A numerical simulator developed by our group [20] using the finite-difference timedomain (FDTD) $[21,22]$ is modified.

In this paper, the cross-borehole pulse radar operated in a well-suited tunnel test site is represented by 3-dimensional model and the finite-difference time-domain (FDTD) method is applied to the model for numerical analysis. Two separate water-filled boreholes and an empty tunnel with cross-section of $2.1 \mathrm{~m}$ by $2.1 \mathrm{~m}$ are surrounded by underground rock, lossy and dispersive granite. Two dipole antennas immersed in the bottom of two water-filled boreholes are moving upwardly with a uniform distance step. At every depth, the radiated pulse from a transmitting antenna is propagated through the granite and picked by a receiving antenna. By stacking picked pulses at every depth continually, B-scan images are obtained. Numerical simulations are repeated by changing the height of collected water from the bottom to the half height of the tunnel with a uniform interval.

Conventional signatures, the time of peak (TOP) and the time of arrival (TOA), are extracted from calculated B-scan images. Fast arrival of the TOP and TOA, the feature of an empty tunnel, is delayed gradually as the height of water inside the tunnel increases. However, the weak TOP and TOA may not be considered as tunnel signatures because those signatures do not meet the accumulating experience of field experiments related with an empty tunnel. Thus, one may be confused whether the delayed TOP and TOA are tunnel signatures or not. To enhance slowed TOP like the TOP of an empty tunnel, a relation curve is derived using measurable parameters of the fastest TOP and the fastest TOA. By adding the calculated enhancement amount obtained from the relation curve, delayed TOP can be improved similar to the TOP of an empty tunnel. Finally, the relation curve is unified by considering two additional granites which cover the low and high dielectric properties of granite.
In the following section, the set-up of 3-dimensional numerical model representing the operation of cross-borehole pulse radar in a well-suited test site in Korea is described. In Section 3, numerical simulations are performed and Bscan images of partially water-filled tunnels are obtained. Then, conventional signatures extracted from B-scan images are interpreted. In Section 4, a relation curve to enhance weak conventional signature is derived. The effects of two additional granites with the low and high dielectric properties on the relation curve are treated properly. Finally, conclusions are summarized in the last section.

\section{Numerical Modeling}

Exploration of a deeply located subversive intrusion tunnel using cross-borehole pulse radar in a well-suited test site is depicted in Figure 1. An empty tunnel with cross-section about $2.1 \mathrm{~m}$ by $2.1 \mathrm{~m}$ is located at $73 \mathrm{~m}$ deep. Two water-filled boreholes are separated by $15.9 \mathrm{~m}$. A transmitting antenna, inserted near the bottom of the left borehole, radiates an electromagnetic pulse. The pulse waveform distorted by the tunnel and underground rock is picked out by a receiving antenna at the same depth in the right borehole. Described measurement is performed until more than $5 \mathrm{~m}$ below the air and water surface in the boreholes by pulling up both antennas simultaneously.

Three-dimensional numerical model of the cross-borehole pulse radar in a well-suited test site is shown in Figure 2(a). In our numerical model, the spatial resolution $(\Delta=\Delta x=\Delta y=\Delta z)$ is uniformly taken by $0.03 \mathrm{~m}$ by considering the electrical property of water and the pulse width of the excited pulse, $10 \mathrm{~ns}$. The relative complex permittivity of water is expressed by Debye's formula [23] as

$$
\varepsilon_{r}(\omega)=\left(\varepsilon_{r \infty}+\frac{\varepsilon_{r s}-\varepsilon_{r \infty}}{1+j \omega \tau}\right)+\frac{\sigma}{j \omega \varepsilon_{0}},
$$

where $\varepsilon_{r \infty}$ is the high frequency permittivity, $\varepsilon_{r s}$ is the zero frequency permittivity, $\tau$ is the relaxation time, $\sigma$ is 


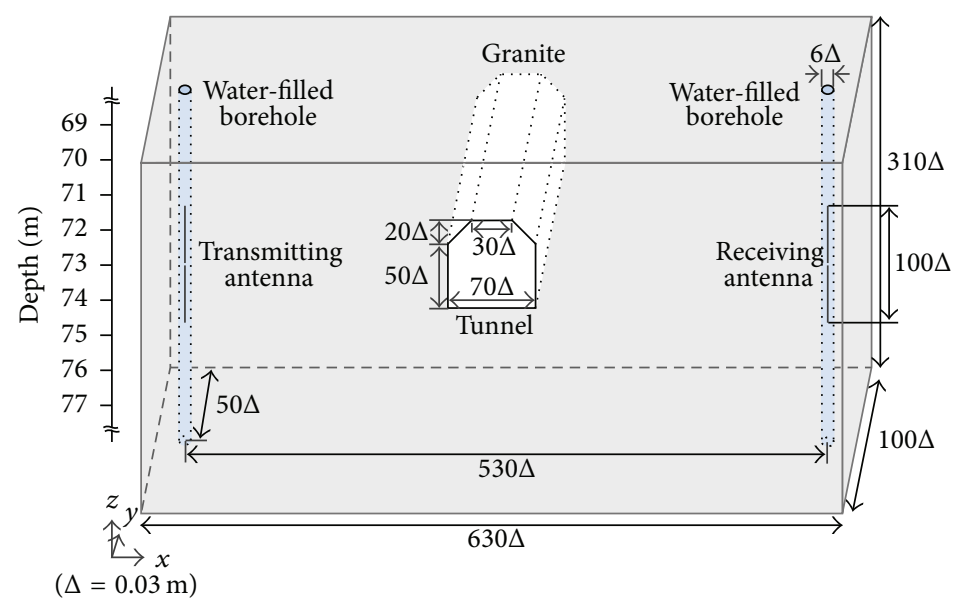

(a)

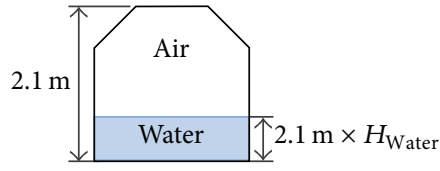

(b)

FIGURE 2: Numerical model of a cross-borehole pulse radar in a well-suited tunnel test site in Korea. (a) Three-dimensional numerical model. (b) Height of collected water inside the tunnel expressed using the height ratio of water $H_{\text {Water }}$ as $2.1 \mathrm{~m} \times H_{\text {Water }}$.

the conductivity, and $\varepsilon_{0}$ is the permittivity in free space. The corresponding parameters of water are $\varepsilon_{r \infty}=5.2, \varepsilon_{r s}=$

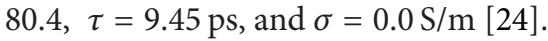

The center of an empty tunnel perpendicularly crossing the vertical plane connecting two boreholes is located at a depth of $73 \mathrm{~m}$. The arch-shaped upper boundary of the tunnel is approximated into a trapezoid. The width and height of the rectangle are $2.1 \mathrm{~m}(70 \Delta)$ and $1.5 \mathrm{~m}(50 \Delta)$, respectively. And the height of the trapezoid is $0.6 \mathrm{~m}(20 \Delta)$. Hence, the total width and height of the tunnel cross-section are $2.1 \mathrm{~m}(70 \Delta)$ and $2.1 \mathrm{~m}(70 \Delta)$, respectively. Proportional to the total height of the tunnel, the height of collected water inside the tunnel is expressed using the height ratio of water inside a tunnel $H_{\text {Water }}$ as $2.1 \mathrm{~m} \times H_{\text {Water }}$ as illustrated in Figure 2(b). The tunnel is infinitely stretched along the $y$-axis. Two boreholes and an empty tunnel are surrounded by a lossy and dispersive rock, granite. The relative complex permittivity of granite is given by Debye's formula with $\varepsilon_{r \infty}=3, \varepsilon_{r s}=9, \tau=2.0 \mathrm{ps}$, and $\sigma=0.0032 \mathrm{~S} / \mathrm{m}$ [7].

Two boreholes with diameter of $0.18 \mathrm{~m}(6 \Delta)$ are separated with the distance of $15.9 \mathrm{~m}(530 \Delta)$ and filled with water. Two dipole antennas separately enclosing transmitter and receiver are immersed in two different water-filled boreholes. The radius and height of the antennas are $0.03 \mathrm{~m}(\Delta)$ and $3 \mathrm{~m}$ $(100 \Delta)$, respectively. And the center of the antennas, feeder region, is enclosed by Teflon $\left(\varepsilon_{r}=2.01\right)$. Each antenna is suspended by a cable which connects the antenna with a ground controller consisting of a computer, a winch, and a power supply. To reduce computational burden, two antennas are winding up only from the depth of $77.75 \mathrm{~m}$ to $68.25 \mathrm{~m}$ with uniform distance interval of $0.15 \mathrm{~m}$. In each depth, the transmitting antenna excites a Gaussian-shaped electromagnetic pulse with the width of 10 ns towards underground rock by employing the one-cell gap model [25]. And the receiving antenna in the other borehole collects the propagated pulse in the same depth. The time step $(\Delta t)$ is chosen by $51.9615 \mathrm{ps}$ to satisfy the stability condition $[21,22]$. The width, length, and height of the 3-dimensional model are $18.9 \mathrm{~m}(630 \Delta)$, $3.0 \mathrm{~m}(100 \Delta)$, and $9.3 \mathrm{~m}(310 \Delta)$, respectively. Finally, as an absorbing boundary condition, the convolutional perfectly matched layer (CPML) [26] is set in the outer region of the 3-dimensional model.

\section{Conventional Tunnel Signatures}

3.1. Time of Peak. FDTD simulations are performed for no tunnel, an empty tunnel, and a partially water-filled tunnel, respectively. In general, an empty tunnel is not fully filled with water since natural drainage is considered during the construction of man-made intrusive tunnel. Hence, half height of the tunnel $\left(H_{\text {Water }}=0.5\right)$ is selected as the height of collected water inside an empty tunnel. Calculated 3 different B-scan images are displayed in Figure 3. The received waveforms of no tunnel in Figure 3(a) are uniform. Thus, the first positive peaks commonly arriving at 280.4086 ns are used as a reference. The B-scan image of the empty tunnel in Figure 3(b) illustrates faster arrival of the first positive peak near the depth of $73 \mathrm{~m}$ than $280.4086 \mathrm{~ns}$ because excited pulse propagates relatively faster through the air region inside a tunnel than the underground rock. The B-scan image of a partially water-filled tunnel in Figure 3(c) also displays faster arrival of the first positive peak near the depth of $73 \mathrm{~m}$ than $280.4086 \mathrm{~ns}$. However, the fast arrival of the first positive peak slows down considerably compared with that of the empty tunnel. It implies that the signature of an empty tunnel, the fast arrival of the first positive peaks, is affected by collected water inside an empty tunnel.

To find the effects of the stagnant water inside the tunnel on tunnel signatures precisely, additional FDTD simulations are performed for 4 different height ratios of water inside the tunnel $\left(H_{\text {Water }}\right), 0.1,0.2,0.3$, and 0.4 , respectively. Then, the most important tunnel signature, the time of peak (TOP), is interpreted. At every depth, the arrival time of the first positive peak is extracted from 6 different B-scan 


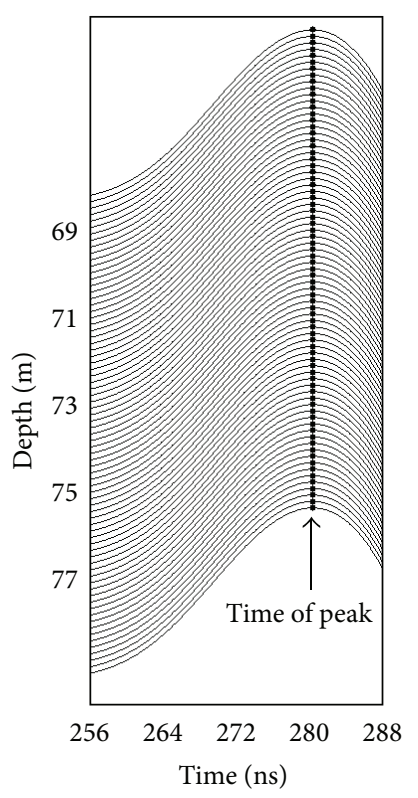

(a)

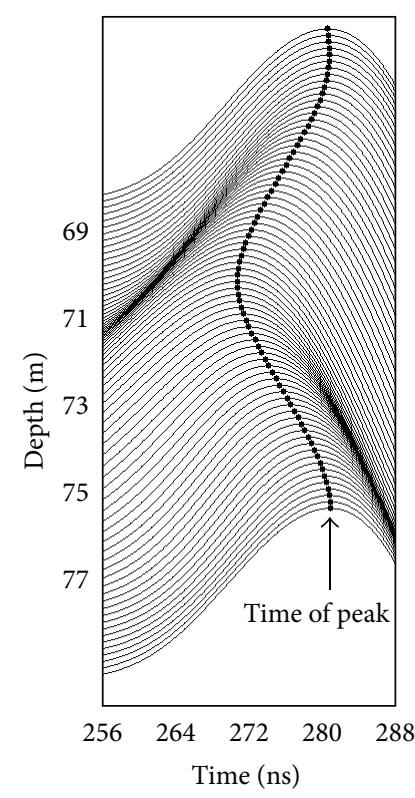

(b)

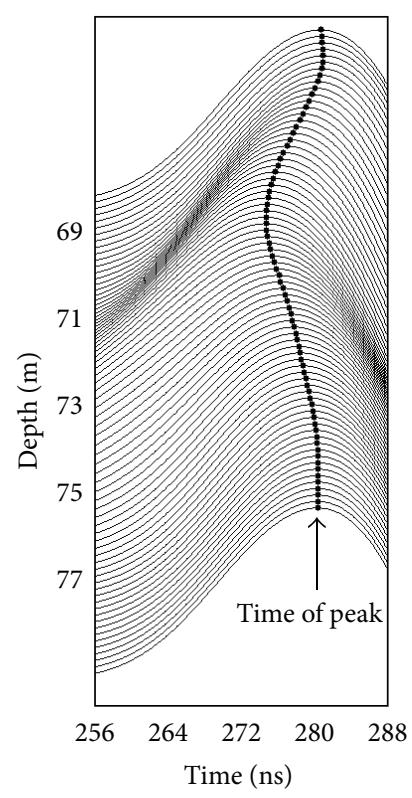

(c)

FIGURE 3: B-scan images computed by applying the FDTD method to the numerical model. (a) No tunnel. (b) Empty tunnel ( $\left.H_{\text {Water }}=0.0\right)$. (c) Partially water-filled tunnel $\left(H_{\text {Water }}=0.5\right)$.

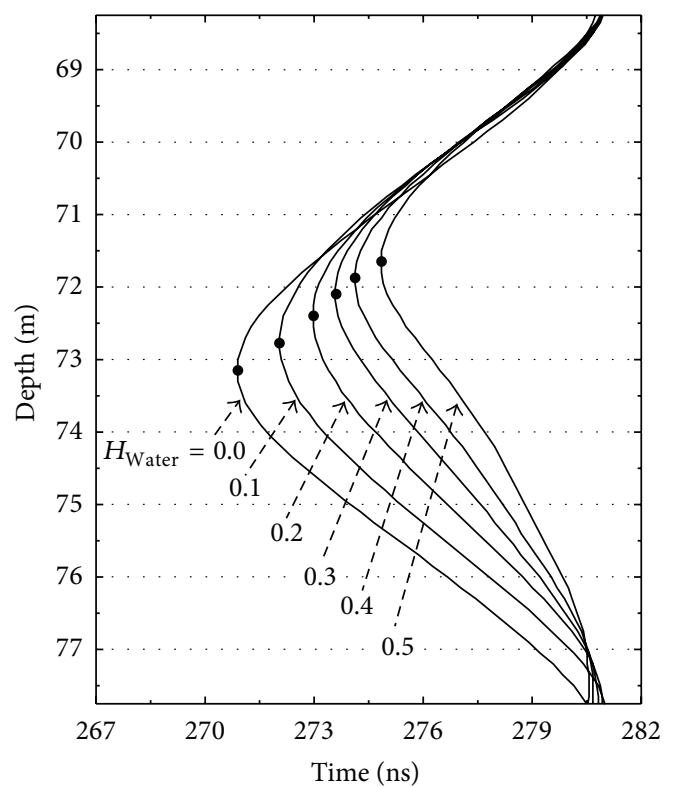

FIGURE 4: The time of peak (TOP) profiles extracted from the Bscan images for 6 different height ratios of water inside the tunnel $\left(H_{\text {Water }}\right)$. The fastest arrival of each TOP profile is marked by a block dot.

images and illustrated in Figure 4. In Figure 4, the fastest arrival of TOP profiles marked by a black dot is gradually delayed depending on the increased height ratio of water in a tunnel $H_{\text {Water }}$. The fastest arrival of each TOP profile corresponding from $H_{\text {Water }}=0.0$ to $H_{\text {Water }}=$ 0.5 is $270.9008 \mathrm{~ns}, 272.0438 \mathrm{~ns}, 272.9790 \mathrm{~ns}, 273.6025 \mathrm{~ns}$,
$274.1220 \mathrm{~ns}$, and $274.8494 \mathrm{~ns}$, respectively. The cause of the delay can be explained by the growing effect of the water in the tunnel which renders the propagation of pulse to be slowed down. In practice, however, the weak TOP which does not meet the accumulating experience of field experiments related with an empty tunnel may not be considered as a tunnel signature. The delayed TOP means the existence of a small tunnel but the cross-section of man-made tunnel is about $2.1 \mathrm{~m}$ by $2.1 \mathrm{~m}$ in general owing to the movement of people through the tunnel. Moreover, information related with the inner state of the tunnel is not provided in advance. Thus, the chance to explore a tunnel may be decreased if the tunnel is partially filled with water. As a detour, another conventional signature which may not be affected severely by collected water in a tunnel needs to be interpreted.

3.2. Time of Arrival. On behalf of the TOP, the time of arrival (TOA) is adopted since the TOA is only related with pulse dominantly propagated through the fastest route inside the tunnel, air $[13,14]$. To find the optimum amplitude level of the TOA at first, the B-scan image of a half water-filled tunnel is considered again. At every depth, time corresponding to the $10 \%, 1 \%$, and $0.1 \%$ of the amplitude of the first positive peak is selected as the TOA and illustrated in Figure 5, respectively. When the amplitude level is $0.1 \%$ of the amplitude of the first positive peak, the fast arrival of the TOA is the most accelerated compared with two amplitude levels of $10 \%$ and $1 \%$. Despite the described advantage, the low amplitude level may be difficult to detect in practice since the amplitude may be smaller than the detectable range of receiving system. The TOA profile with $0.5 \%$ amplitude level of the measured first positive peak utilizing the developed cross-borehole 


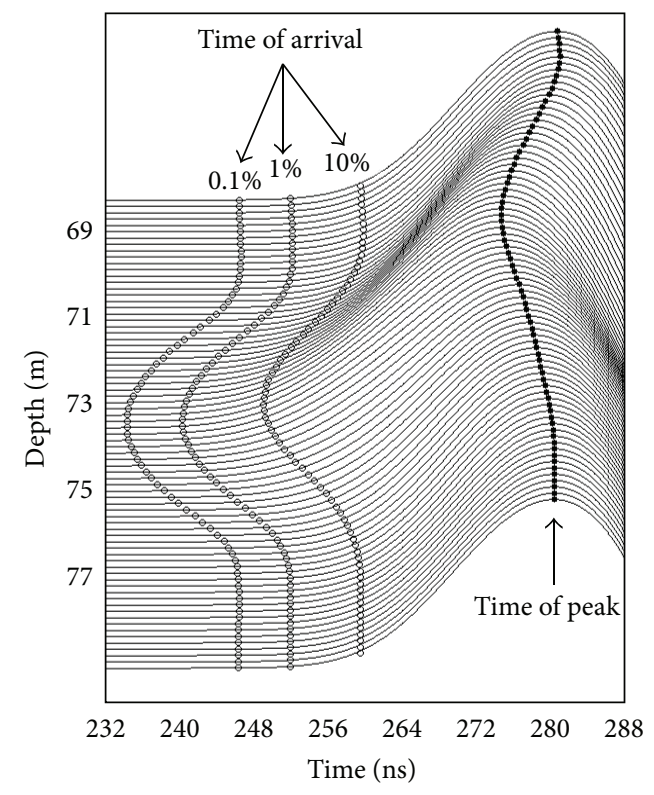

FIGURE 5: B-scan image of a partially water-filled tunnel $\left(H_{\text {Water }}=\right.$ 0.5 ) and the time of arrival with 3 different amplitude levels of $10 \%$, $1 \%$, and $0.1 \%$.

pulse radar in our group was seriously contaminated by noise. However, the much clear TOA profiles were obtained when the amplitude level was increased to $1 \%, 5 \%$, and $10 \%$, respectively [14]. Thus, $10 \%$ of the amplitude level of the first positive peak is considered next. The amplitude level seems to be enough to detect using the receiving system but the pattern of the TOA profile approaches that of the TOP profile. By considering both the fast arrival and the detectable range of receiving system, $1 \%$ of the amplitude of the first positive peak is selected as the optimum amplitude level. The TOA profiles extracted from 6 different B-scan images with the optimum amplitude level of $1 \%$ are illustrated in Figure 6. As expected, the TOA profiles also arrive fast near the depth of $73 \mathrm{~m}$. The fastest arrival of each TOA profile corresponding from $H_{\text {Water }}=0.0$ to $H_{\text {Water }}=$ 0.5 is 238.5329 ns, 238.5849 ns, 238.8966 ns, 239.2603 ns, 239.7799 ns, and 240.5072 ns, respectively.

To quantify fast arrivals, the time advance is introduced by subtracting 6 different TOA profiles from the TOA profile of no tunnel, respectively. Then, the maximum time advance of the TOA profile $\left(\mathrm{TOA}_{\mathrm{Max}}\right)$ is extracted and illustrated in Figure 7 depending on the height ratio of water in a tunnel $H_{\text {Water }}$. By applying the same method to the TOP profile, the maximum time advance of the TOP profile $\left(\mathrm{TOP}_{\mathrm{Max}}\right)$ is chosen and also plotted in Figure 7 for comparison. In Figure 7, the maximum time advance $\mathrm{TOA}_{\mathrm{Max}}$ is larger than the $\mathrm{TOP}_{\mathrm{Max}}$ even though the height ratio of water inside the tunnel $H_{\text {Water }}$ is the same. In spite of the described advantage, the maximum time advance $\mathrm{TOA}_{\mathrm{Max}}$ also slows down depending on the increased height ratio of water in the tunnel $H_{\text {Water }}$. The cause of the slowed TOA can be explained as the decreased portion of the air region inside the tunnel. Even though the degree of decrement is small, the same problem with the TOP has also arisen from the TOA.

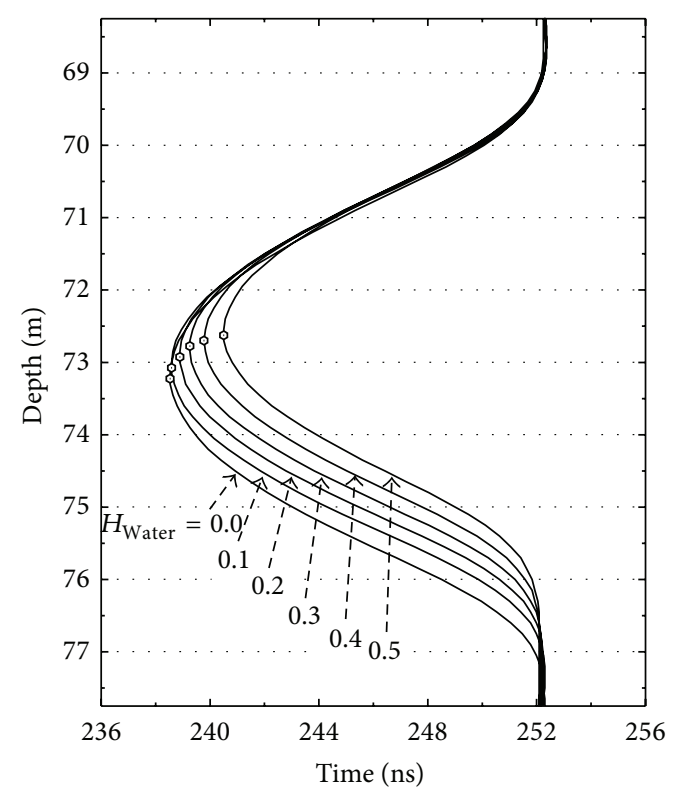

FIgURE 6: The time of arrival (TOA) profiles extracted from the Bscan images for 6 different height ratios of water inside the tunnel $\left(H_{\text {Water }}\right)$ with $1 \%$ amplitude level of the first positive peak. The fastest arrival of each TOA profile is marked by a hollo dot.

\section{Enhanced Tunnel Signature}

4.1. Enhanced Time of Peak. Instead of finding another conventional signature which is robust to the effect of collected water inside an empty tunnel, a method to enhance the weak TOP is investigated. In Figure 7, the delayed maximum time advance $\mathrm{TOP}_{\mathrm{Max}}$ owing to the collected water inside the tunnel can be improved like corresponding value of an empty tunnel $\left(\mathrm{TOP}_{\mathrm{Max}}\left(H_{\text {Water }}=0\right)\right)$ if decreased time advance $T_{\text {Numerical }}$ expressed as

$$
\begin{aligned}
& T_{\text {Numerical }}\left(H_{\text {Water }}\right) \\
& \quad=\operatorname{TOP}_{\text {Max }}\left(H_{\text {Water }}=0\right)-\operatorname{TOP}_{\text {Max }}\left(H_{\text {Water }}\right)
\end{aligned}
$$

can be obtained immediately. Then, the enhanced $\mathrm{TOP}_{\mathrm{Max}}$ $\left(\mathrm{ETOP}_{\mathrm{Max}}\right)$ can be calculated easily by adding the decreased time advance $T_{\text {Numerical }}$ to the measured maximum time advance $\mathrm{TOP}_{\mathrm{Max}}$. However, the decreased time advance $T_{\text {Numerical }}$ cannot be determined immediately using measurable parameter of the maximum time advance $\mathrm{TOP}_{\mathrm{Max}}$ since both the maximum time advance of the empty tunnel $\operatorname{TOP}_{\text {Max }}\left(H_{\text {Water }}=0\right)$ and the height ratio of water in the tunnel $H_{\text {Water }}$ are unknown in practice. Hence, it is very important to find the delayed time advance $T_{\text {Numerical }}$ by employing measurable parameters to enhance the weak TOP.

Thus, a conversion formula is derived to calculate the delayed time advance $T_{\text {Numerical }}$. During the process of formulating, the delayed time advance $T_{\text {Numerical }}$ divided by the maxim time advance $\mathrm{TOP}_{\mathrm{Max}}$, the enhancement rate $T_{\text {Enhance}}$, is utilized to exclude the effect of the dielectric property of 


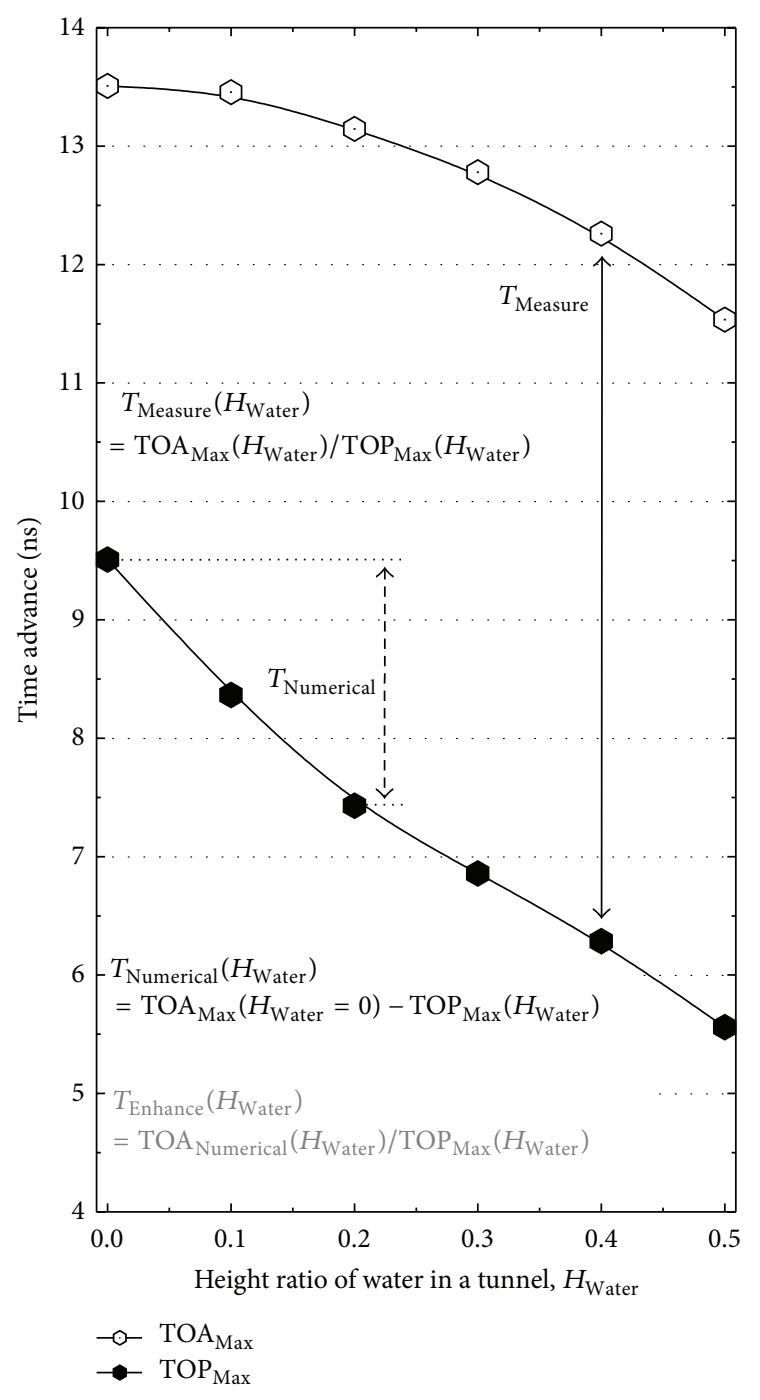

FIgURE 7: Two maximum time advances corresponding to the fastest TOP $\left(\mathrm{TOP}_{\mathrm{Max}}\right)$ and the fastest TOA $\left(\mathrm{TOA}_{\mathrm{Max}}\right)$ for 6 different height ratios of water inside the tunnel $\left(H_{\text {Water }}\right)$.

underground rock. The enhancement rate $T_{\text {Enhance }}$ expressed as

$$
T_{\text {Enhance }}\left(H_{\text {Water }}\right)=\frac{T_{\text {Numerical }}\left(H_{\text {Water }}\right)}{\operatorname{TOP}_{\text {Max }}\left(H_{\text {Water }}\right)},
$$

increases gradually depending on the height ratio of water in the tunnel $H_{\text {Water }}$ since the delayed time advance $T_{\text {Numerical }}$ increases depending on the decreased maximum time advance $\mathrm{TOP}_{\mathrm{Max}}$. If the height ratio of water in the tunnel $H_{\text {Water }}$ can be changed into a measurable parameter, the enhancement rate $T_{\text {Enhance }}$ can be calculated immediately. Thus, measurable rate $T_{\text {Measure }}$ is defined as

$$
T_{\text {Measure }}\left(H_{\text {Water }}\right)=\frac{\operatorname{TOA}_{\text {Max }}\left(H_{\text {Water }}\right)}{\operatorname{TOP}_{\text {Max }}\left(H_{\text {Water }}\right)} .
$$

The measurable rate $T_{\text {Measure }}$ also increases monotonically depending on the height ratio of water in the tunnel $H_{\text {Water }}$

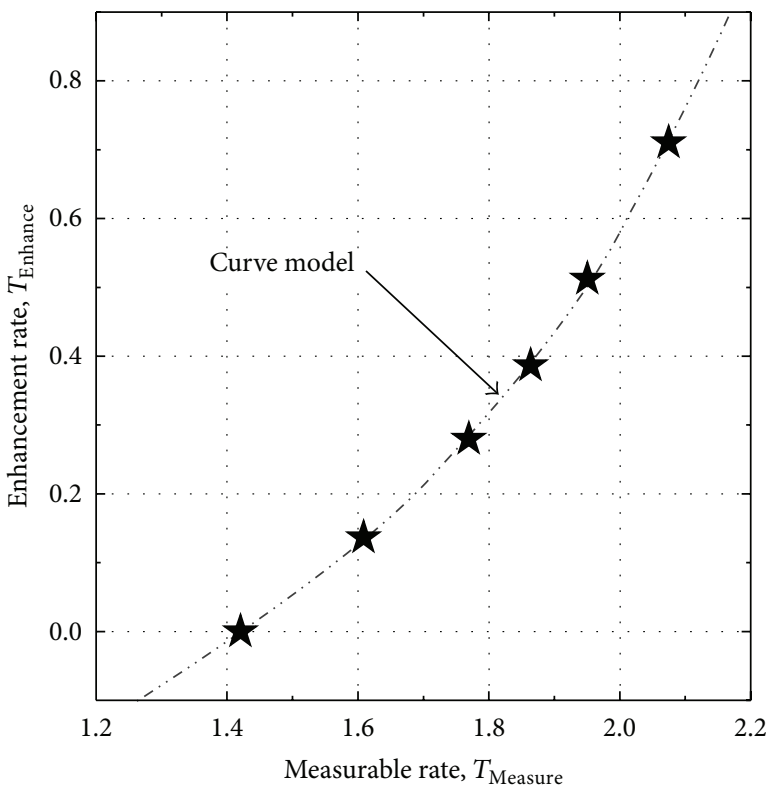

Figure 8: Relation and a curve model to obtain the enhancement rate $T_{\text {Enhance }}$ according to the measurable rate $T_{\text {Measure }}$.

since decrease in the maximum time advance $\mathrm{TOP}_{\mathrm{Max}}$ is larger than the maximum time advance $\mathrm{TOA}_{\mathrm{Max}}$. Thus, oneto-one correspondence between the measurable rate $T_{\text {Measure }}$ and the enhancement rate $T_{\text {Enhance }}$ is illustrated in Figure 8 instead of the height ratio of water in the tunnel $H_{\text {Water }}$. The relation between the $T_{\text {Measure }}$ and $T_{\text {Enhance }}$ displayed in Figure 8 is approximated by a curve of the third-order polynomials as

$$
\begin{aligned}
T_{\text {Enhance }}\left(T_{\text {Measure }}\right)= & -2.5906+4.4501 \times T_{\text {Measure }} \\
& -2.8715 \times T_{\text {Measure }}^{2}+0.7199 \times T_{\text {Measure }}^{3} .
\end{aligned}
$$

Finally, the enhanced maximum time advance ETOP $_{\mathrm{Max}}$ can be calculated utilizing the developed relation curve in (5) and measurable parameters $\mathrm{TOP}_{\mathrm{Max}}$ and $T_{\text {Measure }}$ as

$$
\begin{aligned}
& \operatorname{ETOP}_{\text {Max }}\left(T_{\text {Measure }}\right) \\
& \quad=\operatorname{TOP}_{\text {Max }}+T_{\text {Enhance }}\left(T_{\text {Measure }}\right) \times \operatorname{TOP}_{\text {Max }} .
\end{aligned}
$$

The possibility of the proposed cubic curve is confirmed by comparing the maximum time advances $\mathrm{TOP}_{\mathrm{Max}}$, $\mathrm{TOA}_{\mathrm{Max}}$, and $\mathrm{ETOP}_{\mathrm{Max}}$. To compare the degree of variation relatively, the maximum time advances $\mathrm{TOP}_{\mathrm{Max}}$ and $\mathrm{TOA}_{\mathrm{Max}}$ are divided by corresponding value of an empty tunnel $\operatorname{TOP}_{\text {Max }}\left(H_{\text {Water }}=0\right)$ and $\operatorname{TOA}_{\text {Max }}\left(H_{\text {Water }}=0\right)$ and plotted in Figure 9. The enhanced maximum time advance ETOP $_{\text {Max }}$ is divided by the value of $\operatorname{TOA}_{\text {Max }}\left(H_{\text {Water }}=0\right)$ and also illustrated in Figure 9. Based on the relative time advance of 1.0 , the computed average differences corresponding to the maximum time advances $\mathrm{TOP}_{\mathrm{Max}}, \mathrm{TOA}_{\mathrm{Max}}$, and $\mathrm{ETOP}_{\mathrm{Max}}$ are $22.86 \%, 5.38 \%$, and $0.16 \%$. Different from gradually decreased $\mathrm{TOP}_{\text {Max }}$ and $\mathrm{TOA}_{\text {Max }}$, $\mathrm{ETOP}_{\text {Max }}$ keeps similar 


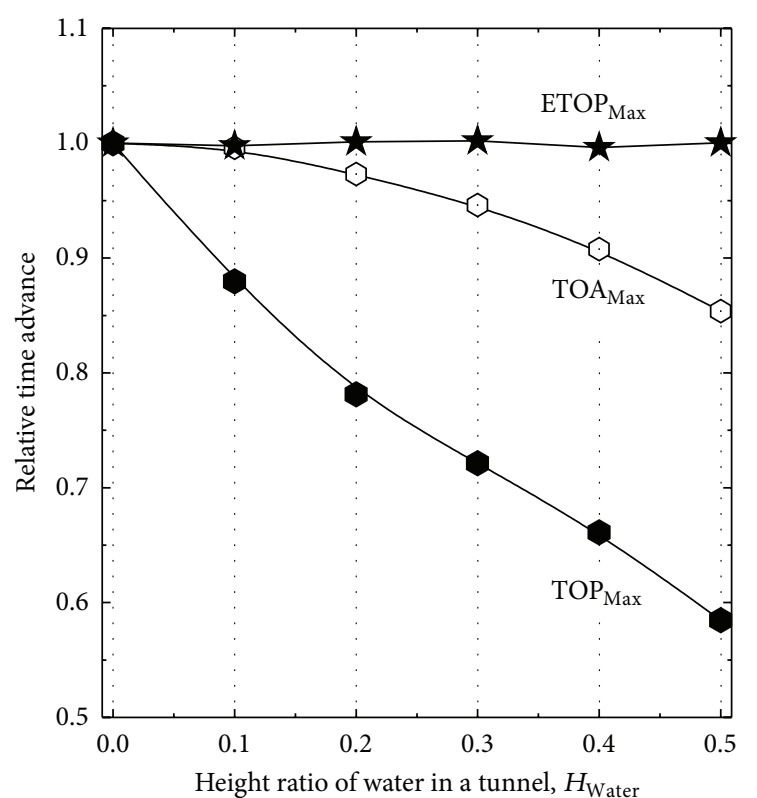

FIGURE 9: Relative time advance corresponding to the fastest TOP $\left(\mathrm{TOP}_{\mathrm{Max}}\right)$, the fastest TOA $\left(\mathrm{TOA}_{\mathrm{Max}}\right)$, and the fastest TOP with enhancement $\left(\right.$ ETOP $\left._{\text {Max }}\right)$.

TABLE 1: Low and high dielectric properties of granite in Korea.

\begin{tabular}{lcccc}
\hline & $\varepsilon_{r s}$ & $\varepsilon_{r \infty}$ & $\tau(\mathrm{ps})$ & $\sigma(\mathrm{S} / \mathrm{m})$ \\
\hline Low dielectric property & 5.8 & 3.0 & 2.0 & 0.001 \\
High dielectric property & 12.0 & 3.0 & 2.0 & 0.005 \\
\hline
\end{tabular}

relative time advance to the value of an empty tunnel even though the $H_{\text {Water }}$ increases.

\subsection{Effects of a Wide Variety of Granites on the Enhanced Time} of Peak. One may suppose that developed curve model to enhance the maximum time advance $\mathrm{TOP}_{\mathrm{Max}}$ may be affected by measurement errors caused during field experiments since the relation curve is introduced from simulation results. However, measurement error caused by receiving system can be ignored since the amplitude of the TOP is large enough compared to the receiver noise [14]. In contrast, measurement error that has arisen from the inhomogeneous underground rock of granite may not be ignored since the dielectric property of granite in Korea suffers from severe variations [15]. It means that developed formula in (5) may be affected dominantly by the various dielectric properties of granite during field experiments in real sites. To cover the effect of inhomogeneous underground rock, simulations are repeated by considering the lower and higher dielectric properties of granite [15] than the medium dielectric properties of granite employed in Section 2. Parameters related with frequency dependent low and high dielectric properties of granite are summarized in Table 1.

The maximum time advances $\mathrm{TOP}_{\mathrm{Max}}$ and $\mathrm{TOA}_{\mathrm{Max}}$ corresponding to the high and low dielectric properties of granites are extracted from calculated B-scan images

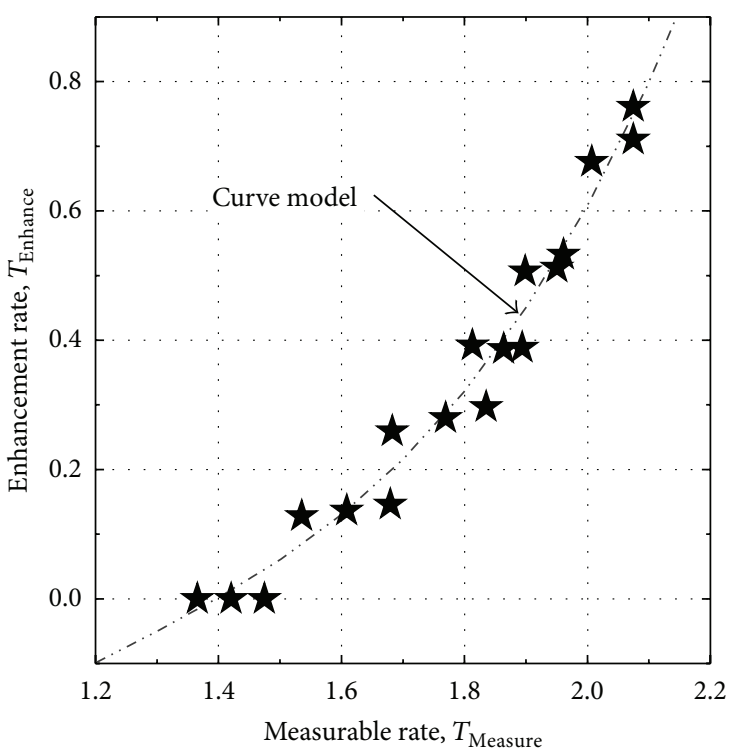

FIGURE 10: Relation and a unified curve model to obtain the enhancement rate $T_{\text {Enhance }}$ according to the measurable rate $T_{\text {Measure }}$ by considering the low, medium, and high dielectric properties of granite.

and listed in Table 2. Then, the measurable rate $T_{\text {Measure }}$ and the enhancement rate $T_{\text {Enhance }}$ are calculated using the values in Table 2. By considering the low, medium, and high dielectric properties of granite together, relations between the measurable rate $T_{\text {Measure }}$ and the enhancement rate $T_{\text {Enhance }}$ are plotted in Figure 10. As a result, a unified relation curve can also be approximated by a cubic relation as

$$
\begin{aligned}
T_{\text {Enhance }}\left(T_{\text {Measure }}\right)= & -2.0493+3.7902 \times T_{\text {Measure }} \\
& -2.6644 \times T_{\text {Measure }}^{2}+0.7168 \times T_{\text {Measure }}^{3} .
\end{aligned}
$$

The possibility of the relation curve in (7) is confirmed in Figure 11 by comparing the relative maximum time advances $\mathrm{TOP}_{\text {Max }}, \mathrm{TOA}_{\mathrm{Max}}$, and $\mathrm{ETOP}_{\mathrm{Max}}$ again. The maximum time advances $\mathrm{TOP}_{\mathrm{Max}}$ and $\mathrm{TOA}_{\mathrm{Max}}$ are divided by corresponding value of an empty tunnel $\mathrm{TOP}_{\text {Max }}\left(H_{\text {Water }}=0\right)$ and $\mathrm{TOA}_{\mathrm{Max}}\left(H_{\text {Water }}=0\right)$. And the enhanced maximum time advance ETOP ${ }_{\text {Max }}$ is divided by the value of $\operatorname{TOP}_{\text {Max }}\left(H_{\text {Water }}=\right.$ $0)$. In Figure 11, small variations are observed at enhanced maximum time advance $\mathrm{ETOP}_{\mathrm{Max}}$ corresponding to the medium dielectric granite. In the cases of the high and low dielectric properties of granite, relatively larger variation seems to be observed at enhanced maximum time ETOP $_{\text {Max }}$ compared to the maximum time $\mathrm{TOA}_{\mathrm{Max}}$ when the height ratio of water in tunnel $H_{\text {Water }}$ is smaller than 0.3. Thus, average differences between relative time advance of 1.0 and the maximum time advances $\mathrm{TOP}_{\mathrm{Max}}, \mathrm{TOA}_{\mathrm{Max}}$, and $\mathrm{ETOP}_{\mathrm{Max}}$ are computed. The computed average differences corresponding to the maximum time advances $\mathrm{TOP}_{\mathrm{Max}}, \mathrm{TOA}_{\mathrm{Max}}$, and ETOP $_{\text {Max }}$ are $22.92 \%, 5.82 \%$, and $2.59 \%$ based on the relative time advance of 1.0. It leads us to conclude that developed 
TABLE 2: The fastest TOP $\left(\mathrm{TOP}_{\mathrm{Max}}\right)$ and the fastest TOA $\left(\mathrm{TOA}_{\mathrm{Max}}\right)$ for 6 different height ratios of water in a tunnel $\left(H_{\text {Water }}\right)$ by considering the low and high dielectric properties of granite.

\begin{tabular}{|c|c|c|c|c|}
\hline \multirow{2}{*}{$H_{\text {Water }}$} & \multicolumn{2}{|c|}{ High dielectric granite } & \multicolumn{2}{|c|}{ Low dielectric granite } \\
\hline & $\mathrm{TOP}_{\mathrm{Max}}(\mathrm{ns})$ & $\mathrm{TOA}_{\mathrm{Max}}(\mathrm{ns})$ & $\mathrm{TOP}_{\mathrm{Max}}(\mathrm{ns})$ & $\mathrm{TOA}_{\mathrm{Max}}(\mathrm{ns})$ \\
\hline 0.0 & 12.3653 & 16.8853 & 6.1307 & 9.0401 \\
\hline 0.1 & 10.9625 & 16.8334 & 5.3514 & 8.9882 \\
\hline 0.2 & 9.8195 & 16.5217 & 4.7279 & 8.6764 \\
\hline 0.3 & 8.8843 & 16.1060 & 4.4162 & 8.3647 \\
\hline 0.4 & 8.2089 & 15.5865 & 4.0005 & 7.8451 \\
\hline 0.5 & 7.3776 & 14.8072 & 3.4810 & 7.2217 \\
\hline
\end{tabular}

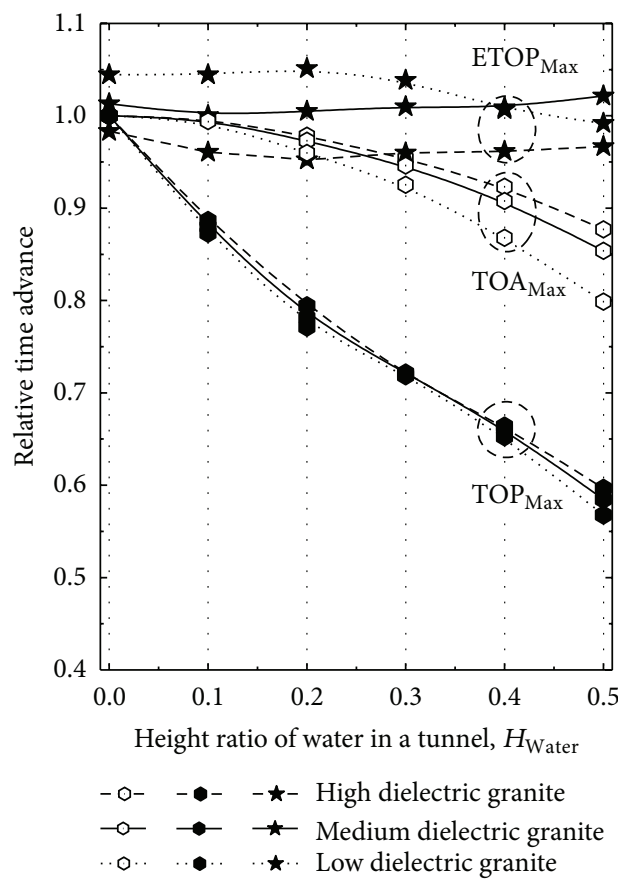

FIGURE 11: Relative time advance corresponding to the fastest TOP $\left(\mathrm{TOP}_{\mathrm{Max}}\right)$, the fastest TOA $\left(\mathrm{TOA}_{\mathrm{Max}}\right)$, and the fastest TOP with enhancement $\left(\mathrm{ETOP}_{\mathrm{Max}}\right)$ by considering the low, medium, and high dielectric properties of granite.

curve model in (7) can be utilized during field experiments to improve weak $\mathrm{TOP}_{\mathrm{Max}}$ due to stagnant water in tunnel.

\section{Conclusion}

FDTD simulations were performed for 6 different water heights from the bottom to the half height of an empty tunnel to interpret the effects of collected water on conventional signatures used to find an empty tunnel. The fastest arrivals of the TOP and TOA were slowed gradually depending on the increased height of water inside a tunnel. To enhance the weak TOP similar to the TOP of an empty tunnel, a cubic relation was formulated using the fastest TOA divided by the fastest TOP. Then, a unified cubic relation including the effect of two granites with the low and high dielectric properties was derived and the possibility was confirmed. Hence, the developed enhancement process will be helpful for detecting a tunnel in real sites even though the tunnel is partially filled with water.

\section{Conflict of Interests}

The authors declare that there is no conflict of interests regarding the publication of this paper.

\section{Acknowledgment}

This work was supported by the Korea Institute of Science and Technology Institutional Program under Grant 2E24790.

\section{References}

[1] R. J. Lytle, E. F. Laine, D. L. Lager, and D. T. Davis, "Crossborehole electromagnetic probing to locate high-contrast anomalies," Geophysics, vol. 44, no. 10, pp. 1667-1676, 1979.

[2] T.-K. Lee, S.-O. Park, J.-W. Ra, and S.-Y. Kim, "Near-field diffraction pattern by an underground void of circular cylinder," Microwave and Optical Technology Letters, vol. 2, no. 5, pp. 179183, 1989.

[3] J. Schneider, J. Brew, and I. C. Peden, "Electromagnetic detection of buried dielectric targets," IEEE Transactions on Geoscience and Remote Sensing, vol. 29, no. 4, pp. 555-562, 1991.

[4] S.-Y. Kim and J.-W. Ra, "The role of cross borehole radar in the discovery of fourth tunnel at Korea DMZ," in Proceedings of 4th Tunnel Detection Symposium on Subsurface Exploration Technology, pp. 253-257, Golden, Colo, USA, 1993.

[5] H.-K. Choi and J.-W. Ra, "Detection and identification of a tunnel by iterative inversion from cross-borehole CW measurements," Microwave and Optical Technology Letters, vol. 21, no. 6, pp. 458-465, 1999.

[6] I. C. Peden and J. Brew, "A laboratory scale model for the study of subsurface scattering in low-loss media with applications to ground penetrating radar," Journal of Applied Geophysics, vol. 33, no. 1-3, pp. 109-118, 1995.

[7] G. R. Olhoeft, "Interpretation of hole-to-hole radar measurements," in Proceedings of 3rd Technical Symposium on Tunnel Detection, pp. 616-628, Golden, Colo, USA, 1988.

[8] M. L. Moran and R. J. Greenfield, "Radar signature of a 2.5-D tunnel," Geophysics, vol. 58, no. 11, pp. 1573-1587, 1993.

[9] H. Zhou and M. Sato, "Subsurface cavity imaging by crosshole borehole radar measurements," IEEE Transactions on Geoscience and Remote Sensing, vol. 42, no. 2, pp. 335-341, 2004. 
[10] S. Ebihara, "Directional borehole radar with dipole antenna array using optical modulators," IEEE Transactions on Geoscience and Remote Sensing, vol. 42, no. 1, pp. 45-58, 2004.

[11] K. Takahashi and M. Sato, "Parametric inversion technique for location of cylindrical Structures by cross-hole measurements," IEEE Transactions on Geoscience and Remote Sensing, vol. 44, no. 11, pp. 3348-3355, 2006.

[12] H. Zhou, M. Sato, T. Takenaka, and G. Li, "Reconstruction from antenna-transformed radar data using a time-domain reconstruction method," IEEE Transactions on Geoscience and Remote Sensing, vol. 45, no. 3, pp. 689-696, 2007.

[13] S.-W. Kim and S.-Y. Kim, "Analysis of cross-borehole pulse radar signatures measured at various tunnel angles," Exploration Geophysics, vol. 41, no. 1, pp. 96-101, 2010.

[14] S.-W. Kim, S.-Y. Kim, and S. Nam, "Estimation of the penetration angle of a man-made tunnel using time of arrival measured by short-pulse cross-borehole radar," Geophysics, vol. 75, no. 3 , pp. J11-J18, 2010.

[15] S.-W. Kim and S.-Y. Kim, "Estimation of the strike angle of an empty tunnel in different rocks using a cross-borehole pulse radar," Radio Science, vol. 47, no. 1, pp. 1-11, 2012.

[16] M. L. Moran and R. J. Greenfield, "Radar waveforms from a three dimensional tunnel," in Proceedings of the 4th Tunnel Detection Symposium on Subsurface Exploration Technology, pp. 71-84, Golden, Colo, USA, 1993.

[17] G. A. Ellis and I. C. Peden, "Analysis technique for buried inhomogeneous dielectric objects in the presence of an air-earth interface," IEEE Transactions on Geoscience and Remote Sensing, vol. 33, no. 3, pp. 535-540, 1995.

[18] G. Bozza, C. Estatico, M. Pastorino, and A. Randazzo, "Application of an inexact-Newton method within the second-order Born approximation to buried objects," IEEE Geoscience and Remote Sensing Letters, vol. 4, no. 1, pp. 51-55, 2007.

[19] S. Caorsi, A. Massa, M. Pastorino, M. Raffetto, and A. Randazzo, "Detection of buried inhomogeneous elliptic cylinders by a memetic algorithm," IEEE Transactions on Antennas and Propagation, vol. 51, no. 10, pp. 2878-2884, 2003.

[20] J.-H. Jung, S.-W. Kim, Y.-S. Kim, and S.-Y. Kim, "Electromagnetic propagation from the intestine-ingested source in the human body model," IEEE Transactions on Antennas and Propagation, vol. 58, no. 5, pp. 1683-1688, 2010.

[21] K. S. Yee, "Numerical solution of initial boundary value problems involving Maxwell's equations in isotropic media," IEEE Transactions on Antennas and Propagation, vol. 14, no. 3, pp. 302-307, 1966.

[22] A. Taflove and S. C. Hagness, Computational Electrodynamics: Finite-Difference Time-Domain Method, Artech House, Norwood, NJ, USA, 2000.

[23] R. Luebbers, F. P. Hunsberger, K. S. Kunz, R. B. Standler, and M. P. Schneider, "A frequency-dependent finite-difference timedomain formulation for dispersive materials," IEEE Transactions on Electromagnetic Compatibility, vol. 32, no. 3, pp. 222227, 1990.

[24] D. M. Hagl, D. Popovic, S. C. Hagness, J. H. Booske, and M. Okoniewski, "Sensing volume of open-ended coaxial probes for dielectric characterization of breast tissue at microwave frequencies," IEEE Transactions on Microwave Theory and Techniques, vol. 51, no. 4, pp. 1194-1206, 2003.

[25] S.-Y. Hyun, Electromagnetic characterization of impulse groundpenetrating radar [Ph. D. dissertation], Department of Radio Sciences and Engineering, Korea University, Seoul, South Korea, 2008.
[26] J. A. Roden and S. D. Gedney, "Convolutional PML (CPML): an efficient FDTD implementation of the CFS-PML for arbitrary media," Microwave and Optical Technology Letters, vol. 27, pp. 334-339, 2000. 

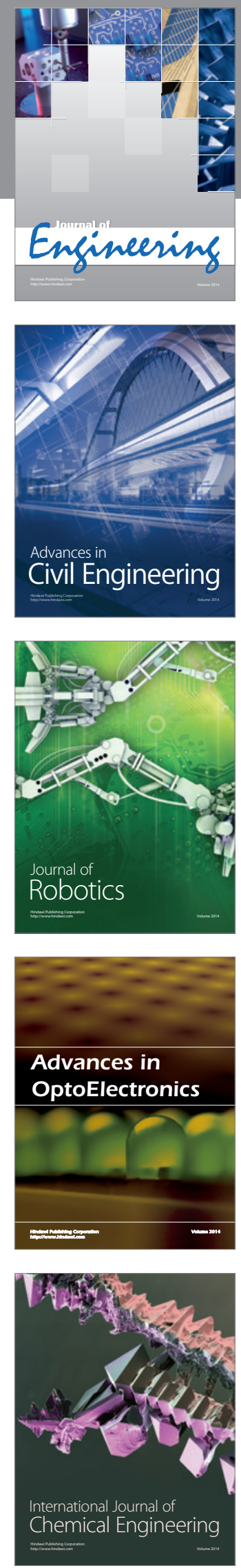

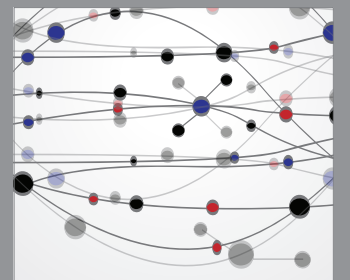

The Scientific World Journal
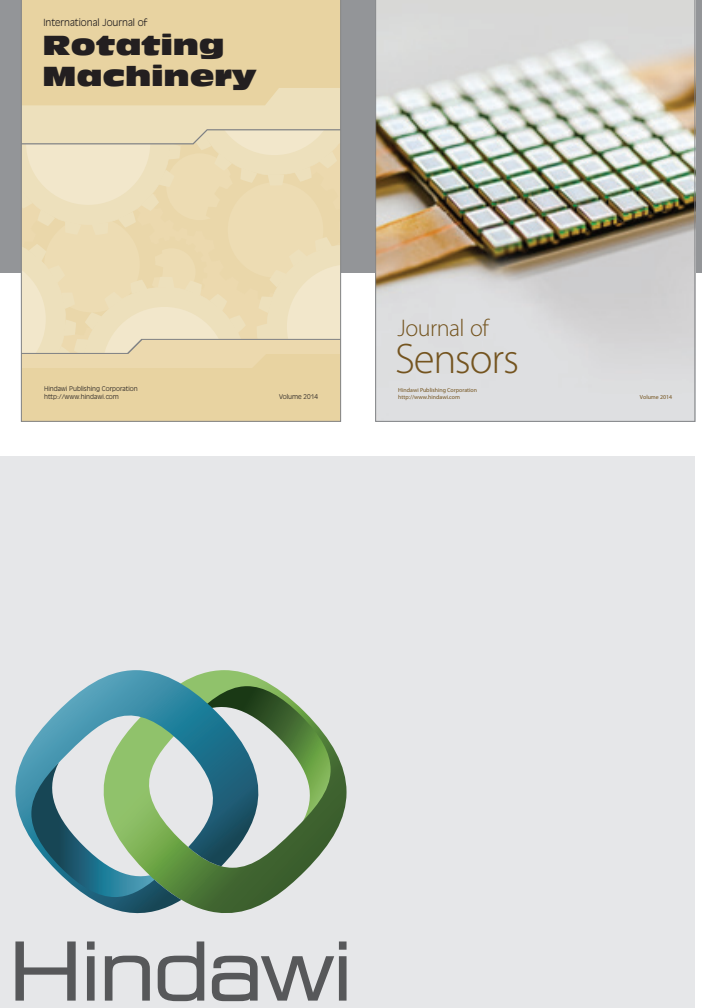

Submit your manuscripts at http://www.hindawi.com
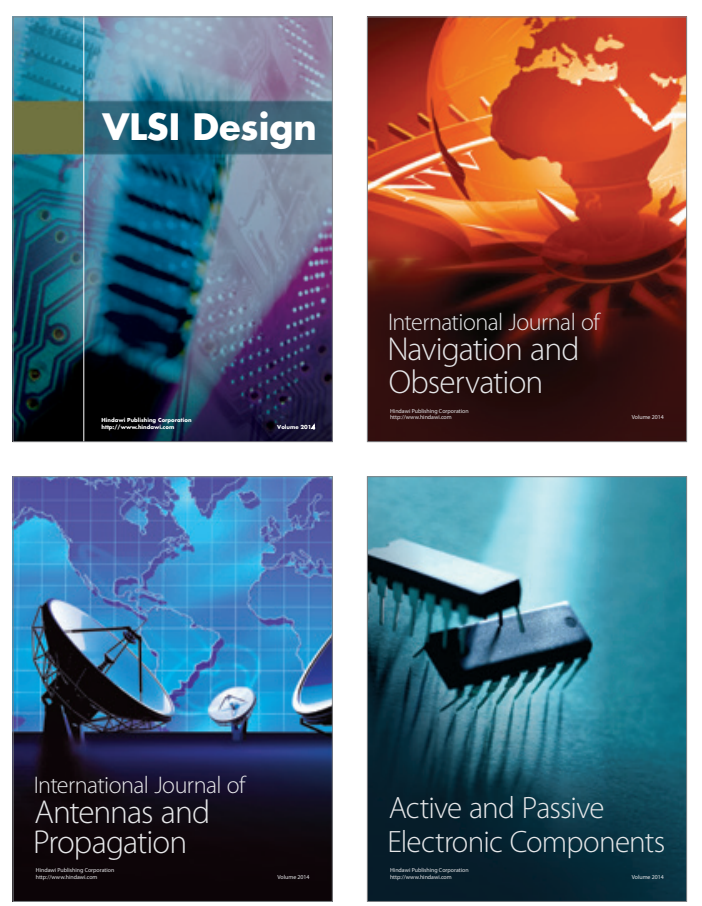
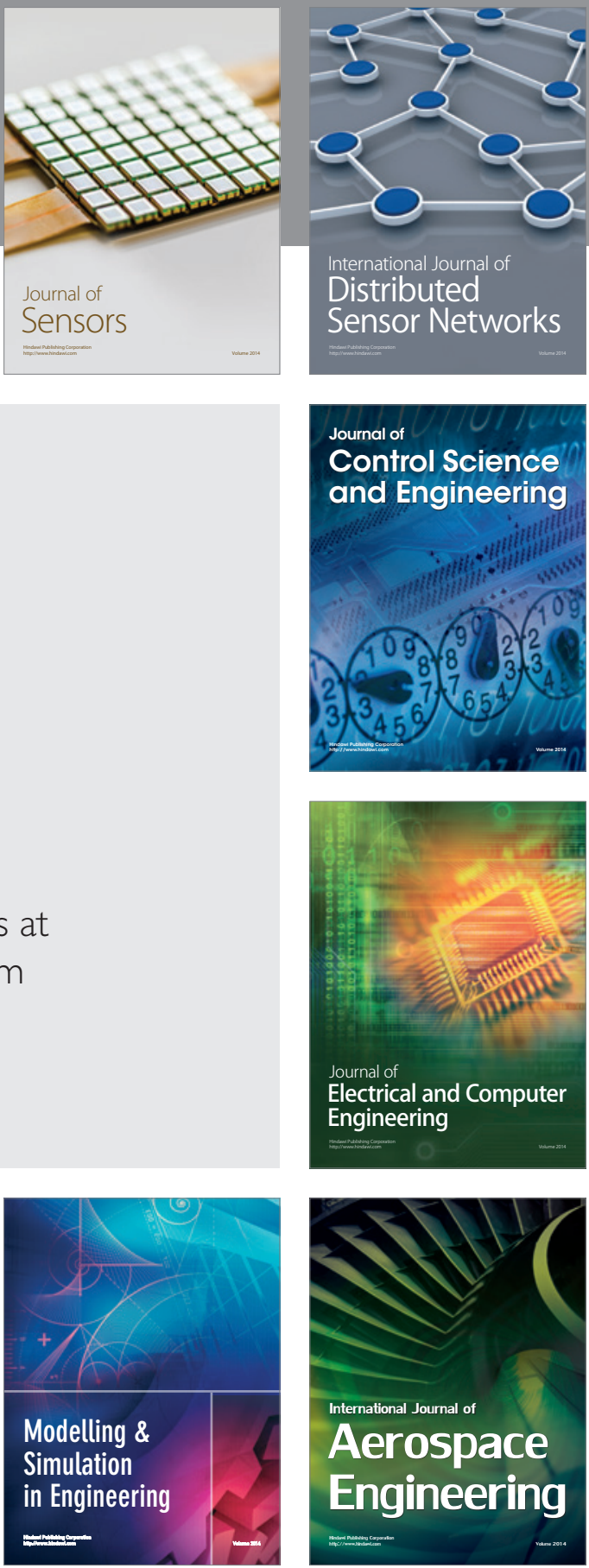

Journal of

Control Science

and Engineering
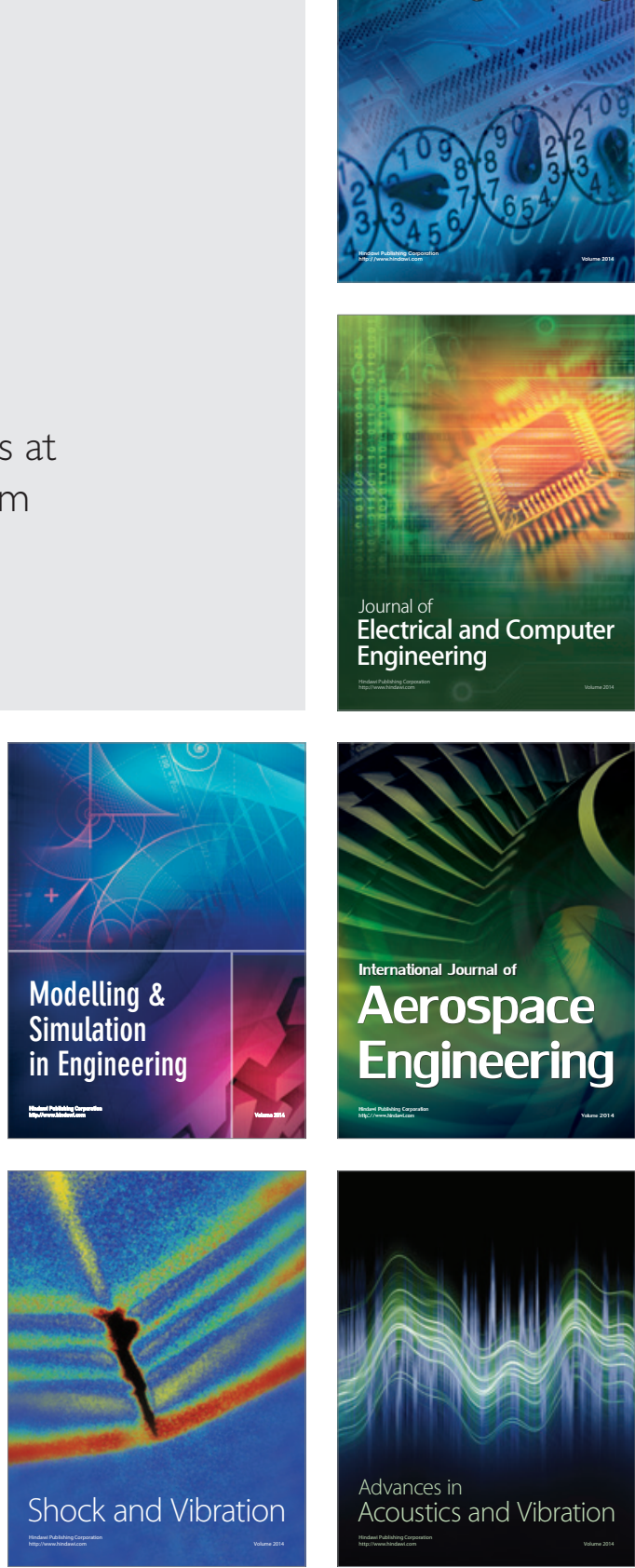